\title{
DESPUÉS DEL FUNDACIONISMO: ENTRE EL CÍRCULO VICIOSO Y EL REGRESO AL INFINITO*
}

\author{
BAS C. van FraAsSEN
}

Universidad de Princeton

Como texto guía deseo citar un pasaje de los Philosophical Papers de Hilary Putnam en el que, de manera indirecta, él mismo cita a Strawson:

Como lo señaló Strawson hace muchos años, constantemente se nos pide que elijamos entre posiciones metafisicas, por una parte, y posiciones reduccionistas por la otra, y, lo que es terriblemente dificil (pero lo que hace que valga la pena el juego filosófico), mostrar que el misterio metafísico no es la única alternativa a la posición simplista del reduccionista (y viceversa, claro está). (Putnam, 1983, p. xvi)

Cito lo anterior para mostrar mi propia forma de acuerdo con el "proyecto fundamental" en filosofía (si se me permite emplear un término sartreano) de Putnam de toda la vida. Claro está que nuestra costumbre y cortesía en filosofía es subrayar lo negativo, insistir en señalar nuestros desacuerdos ( $y$ así, supongo, por implicación, lo distintivo de nuestra propia contribución). Permítaseme, por un momento, superar mi amour propre y precisar cómo me sitúo del mismo lado filosófico que han delimitado los escritos de Putnam.

Las dos Conferencias Howison de Putnam, en Berkeley, en 1981, atacan, primero que nada, la metafísica naturalizada y, en segundo lugar, la epistemología naturalizada. ${ }^{1}$ También éstas son dos de las tendencias generales en contra de las que yo me identifico. Diversos escritos anteriores de Putnam, de los años 60 y 70, diagnosticaron qué es lo que estaba mal con la generación filosófica anterior, como su obsesión con la verificación y, en general, su fundacionismo epistemológico; yo acepto plenamente este diagnóstico. También concuerdo en que la razón no puede naturalizarse y que no puede desterrarse lo normativo sin perder, incluso, el status factual de aseveraciones y creencias como aseveraciones y creencias (distintas de meros fenómenos vocales y

* Este escrito contiene una versión de las primeras tres secciones centrales de mi artículo "From Vicious Circle to Infinite Regress and Back Again", que se presentara como discurso inaugural ante la Philosophy of Science Association en octubre de 1992.

1 Putnam, 1983, caps. 12 y 13. 
subvocales). Finalmente, concuerdo con su aseveración de que cualquier posición que conduzca a la relatividad ontológica, en el sentido de Quine, por esto mismo se reduce al absurdo.

Ciertamente, si trato de encontrar desacuerdos generales que yo quisiera registrar, no puedo dar con ninguna doctrina de Putnam que yo deseara rechazar. En parte, quizás, esto es porque Putnam no es dogmático y siempre está dispuesto a considerar, bajo una nueva luz, sus mismas propuestas anteriores. Parece, entonces, que las diferencias estriban en nuestras corazonadas acerca de cómo mejorar nuestro pasado y dónde podemos esperar avances productivos. Ahí, en general, me encuentro en un estado de ánimo diferente. Sin negar el gran éxito de la filosofía del lenguaje en nuestro siglo, ya no me. parece que el lenguaje, la verdad, la referencia y el significado nos proporcionen un camino dorado o incluso preferible hacia la iluminación filosófica. Demasiada filosofia del lenguaje nos ha llevado por rodeos o desviaciones. Consideremos, digo, como bien aprendidas nuestras lecciones sobre el lenguaje; confiemos plenamente que nos apartarán de trampas metafísicas pasadas; pero no continuemos abordando todas las cuestiones filosóficas en sus términos. Pero, como dije, esto es cuestión de corazonadas. No espero que el profesor Putnam dé argumentos en contra de iuna corazonada!

\section{****}

La filosofía moderna fue una búsqueda de fundamentos; en gran medida, la filosofia contemporánea rechaza esta búsqueda como algo equivocado. No argumentaré aquí en contra del fundacionismo; doy por hecho su rechazo. Hacer esto es, también, dar por hecho que se han demolido ciertas formas de argumentos escépticos. Esto se aplica, particularmente, a argumentos de la forma, "esto o aquello que consideramos racional no puede justificarse en contra de sus posibles rivales; por lo tanto, es arbitrario e irracional". Cualquier argumento semejante se funda en una insistencia en los fundamentos, que doy por hecho que es un error. ${ }^{2}$

Empero, yo creo que aún nos hechizan ciertos espectros de nuestro pasado fundacionista. Cada uno de ellos nos amenaza con un relativismo debilitante. Los espectros que estudiaré están conectados, no tanto por sus similitudes superficiales, sino por mi diagnóstico y por mi solución. Mientras que los susurros de estos espectros son, en general acerca de la muerte de la epistemología, yo, específicamente, cuando pueda hacerlo, relacionaré los problemas con la ciencia. En cada caso, dirigiré nuestra atención a ciertos aspectos pragmáticos

2 De manera alternativa, puede apoyarse en el concepto "prusiano" de racionalidad, que identifica lo que es racional con lo que se impone racionalmente (a diferencia de lo que es racionalmente permitido). Esto tambićn lo rechazo aquí sin argumento: la racionalidad es sollo irracionalidad reprimida. (Véase la discusión con Putnam en Florencia (1978, p. 110, en mi 1984).) 
de la ciencia. La ciencia la concibo como aportándonos modelos. Ahora, en filosofía de la ciencia y en epistemología general, nos es crucial apreciar la autonomía del acto de relacioriamos con esos modelos si hemos de usarlos. En breve, esto significa cierta autonomía de la ciencia aplicada frente a la ciencia pura. Esto implica la necesidad de pensar en términos de la perspectiva de los científicos individuales y de las comunidades científicas y no concentrarse tan sólo en el contenido de sus teorías y creencias.

\section{Espectro I: pérdida de objetividad}

Deseo comenzar con una perplejidad familiar acerca de los mapas. Si estoy perdido y compro un mapa, eso no basta. Normalmente, los mapas no tienen una flecha con una etiqueta "Usted está aque". Pero, incluso, si el mapa que obtengo la tuviese, el problema sería realmente el mismo, esto es, tengo que localizar dónde estoy con respecto a esa flecha. (Imaginemos que encontré ese mapa tirado en la calle. Imaginemos instrucciones acerca del significado de tales flechas etiquetadas: "Si está ante un mapa en condición $C$, entonces..." Usted aún necesita aportar la premisa deíctica, "estoy ante un mapa en condición C".)

La información extra que se requiere para usar el mapa no puede codificarse en ese mapa. Cuando tengo esa información extra puedo expresarla señalando un punto en ese mapa y diciendo, "Estoy ahr" - una autoadscripción de localización sobre el mapa.

También este acto puede describirse y puede incluirse la información de que se efectúa en un mapa mayor (con la etiqueta "localización de la lectura del mapa por parte de $\mathrm{vF}$ en el tiempo $t$ "). Pero entonces, claro está, lo que aún necesito para usar ese mapa es una autoadscripción de localización con respecto a ese mapa. Esto no altera el problema de que, con este nuevo mapa, yo puedo autoadscribir una localización mediante las palabras diferentes, "Yo soy $v F$ y ahora es $t$ ". El intento de reemplazar o eliminar estas autoadscripciones conduce a un regreso al infinito, empleando una serie infinita de mapas. Pero incluso, suponiendo la precisión de toda la serie de mapas, el regreso no logra eliminar la necesidad de la autoadscripción. Pues aún estaré perdido a menos que pueda localizarme con respecto a al menos uno de ellos y esto sólo puedo hacerlo aseverando una autoadscrpción que no es deducible a partir de la precisión de esos mapas.

El tema de la autoadscripción pertenece a la pragmática y no a la semántica. Ésta es una forma caprichosa de decir que lo que hace no puede igualarse con el contenido de un mapa o con la creencia de que cierto mapa "cuadra con" el mundo. 
El alcance de esta perplejidad. Esta propuesta acerca de mapas es un paradigma de la diferencia entre ciencias pura y aplicada. El cuerpo de la ciencia, la totalidad de la información científica aceptada, puede escribirse, en principio, en forma libre de coordenadas, independiente de contexto. Eso le es posible a la ciencia pura, aun si incluye la historia del universo o la evolución de especies biológicas sobre la tierra. Pero, para aplicar este cuerpo de ciencia en tecnología o, incluso, para contrastarlo o usarlo para explicar algo o ampliarlo mediante investigación, el científico o la comunidad científica debe proporcionar algo más que no viene con ese cuerpo de ciencia, pero que sirve para localizar al usuario con respecto a él.

Permítaseme formular esto, de alguna manera un tanto diferente, en términos de modelos. "Modelo" es una metáfora cuya base es el modelo construido sobre la cubierta de una mesa. Usamos esta metáfora cuando hablamos de modelos cosmológicos, modelos de espacios de Hilbert y cosas similares. Podríamos haber usado la palabra "mapa" y hacer de los mapas, igualmente bien, la base de nuestra metáfora.

Ahora supongamos que la ciencia nos da un modelo que putativamente representa el mundo con todo detalle. Supongamos, incluso, que esto es así. Supongamos que consideramos que sabemos que lo es. Aun entonces, antes de que podamos pasar a usar ese modelo, hacer predicciones y construir puentes, debemos localizarnos con respecto a ese modelo. Así que, aparentemente, necesitamos saber algo más, además de lo que aquí nos ha dado la ciencia. Lo adicional es la autoadscripción de localización.

Pero ahora aparece el primer espectro y nos dice que tenemos un dilema. $O$ bien decimos que la autoadscripción es un simple enunciado factual objetivo o decimos que es algo irreductiblemente subjetivo. En el primer caso la ciencia, inevitablemente, está condenada a ser objetivamente incompleta. En el segundo caso, también hemos admitido un límite a la objetividad, hemos dejado entrar la subjetividad a la ciencia.

Ilustración histórica. Carnap se enfrentó a esto en el Aufbau (1928), cuando intentó pensar a fondo su tesis estructuralista acerca de la ciencia. Él comienza la segunda parte del $A u f b a u$ con el anuncio:

mantendremos e intentaremos establecer la tesis de que la ciencia trata soblo con la descripción de las propiedades estructurales de los objetos.

Lo que esto significa es que, en la ciencia teórica, al igual que en la matemática, lo que se describe se describe sólo hasta el isomorfismo. Lo que exactamente produce la ciencia teórica es, ni más ni menos, modelos matemáticos de los 
objetos y los procesos físicos. Y esto, como Carnap lo explica ampliamente, está muy alejado de lo que ordinariamente llamamos una descripción. ${ }^{3}$

Como Platón lo dijo de la poesía y del arte, así Carnap nos dice de la ciencia que está a varios grados de distancia de la realidad, que procede mediante dos etapas de abstracción:

Una descripción de propiedad indica las propiedades que tienen los objetos individuales de un dominio dado, mientras que una descripción de relación indica las relaciones que se dan entre estos objetos, pero no hace ninguna aseveración acerca de los objetos como individuos. (Sec. 10, p. 19)

Hay cierto tipo de descripción de relación que llamaremos descripción de estructura. A diferencia de las descripciones de relación, éstas no sólo dejan sin mencionar las propiedades de los elementos individuaies del campo, ellas ni si quiera especifican las relaciones mismas que se dan entre estos elementos. En una descripción de estructura sólo se indica la estructura de la relación, esto es, la totalidad de sus propiedades formales. (Sec. 11, p. 21)

El problema crucial aparece luego, en la siguiente sección:

Así, nuestra tesis, a saber, que los enunciados científicos se refieren sólo a propiedades estructurales, equivale a la aseveración de que los enunciados científicos hablan sólo de formas sin enunciar qué sean los elementos y las relaciones de estas formas. De manera superficial, ésta parece ser una aseveración paradójica [...] en la ciencia empírica uno debería saber si habla de personas o de poblaciones. Ésta es la propuesta decisiva: la ciencia empirica debe estar en posición de distinguir estas diversas entidades... (Sec. 12, p. 23)

La expresión, "de manera superficial", convierte lo anterior en un enorme eufemismo. Las siguientes siete páginas se usan para desentrañar esa paradoja "superficial".4

He de subrayar que este problema es agudo para cualquier enfoque de la ciencia que caracterice la teoría fisica primariamente en términos de modelos matemáticos. La frase, "modelo matemático", es casi totalmente redundante en este contexto, porque la única alternativa es lo que se podría denominar "modelos de mesa", esto es, estructuras concretas con etiquetas pegadas. Incluso concediendo modelos tanto analógicos como digitales, éstos son tan finitos de tantas maneras que simplemente no nos llevan muy lejos si hoy es-

3 Véase, además, la exploración de esta propuesta en el artículo “Scientific Realism or Irenic Instrumentalism" de Sellars; esta visión de la ciencia también la presentó Russell, por ejemplo en su libro Los problemas de la filosofía.

4 Todas las citas son de la traducción de R.A. George (1967). Véase, además, la página 107 y la paradójica apertura de la sec. 180, p. 290. 
tamos hablando acerca de la ciencia teórica. ${ }^{5}$ Sin embargo, las únicas estructuras abstractas manejables son las que nos da la matemática. Y la descripción matemática es única, a lo más, hasta el isomorfismo. Nosotros podemos pegar etiquetas, pero este "pegar" también tiene, como sentido literal, sólo la existencia de alguna función la que, a su vez, está sujeta a las mismas limitaciones de descripción.

Presumiblemente, este problema hay que enfrentarlo invirtiendo la máxima de Kant de que en cualquier cosa hay tanta ciencia pura como matemáticas haya. ${ }^{6}$ Es decir, podemos convenir en esto cuando se trata de la ciencia pura o de la teoría, pero negar que sea esto todo lo que es importante en la ciencia -por tanto, la ciencia empírica, al ser más que matemática, debe de ser más que teoría pura. Una teoría empírica debe destacar una parte específica del mundo, establecer referencia con esa parte y decir - a manera de aseveración contingente, sustantiva, acerca del mundo- que sus modelos cuadran con eso. Pero, ¿cómo exactamente puede hacerse esto?

El problema con esa pregunta es que su respuesta tiene que ser científicamente respetable y, así, la misma debe considerarse, también, como parte de la ciencia. La tarea de producir esa respuesta científicamente respetable es, exactamente, la que Carnap intenta en las siguientes siete páginas. Veamos a dónde lo conduce.

En la sección 13 dice que hay dos medios, por descripción ostensiva que se apoya en la percepción y en indicaciones mediante gestos, tales como señalar mientras uno dice "Ése es el Mont Blanc" y por descripción definida que, de manera única, distingue el objeto mediante una lista de algunas de sus propiedades. Él admite que parece como si el uso de las descripciones definidas será exitoso sólo si, en última instancia, se apoya en alguna descripción ostensiva. Pero, su reacción ante eso es:

Sin embargo, en un momento veremos que, dentro de cualquier dominio de objetos, en principio es posible un sistema único de descripciones definidas aun sin ayuda de la descripción ostensiva. (Sec. 13, pp. 24-25)

5 Yo objeto fuertemente la asimilación de los modelos a analogías y metáforas (quizás a diferencia de lo inverso), que hacen surgir caracterizaciones tales como "un modelo en ciencia es una analogía sistemática postulada entre un fenómeno cuyas leyes son ya conocidas y el que se investiga". Ciertamente hay tales casos especiales; por ejemplo, se podría decir que, en cierto momento histórico, la difusión de gas era un modelo de la difusión de calor o el sistema solar lo era del átomo. Pero tal analogía es sólo un primer paso; la directiva no es, simplemente, "mirar" al primero; el consejo proferido es comenzar con las ecuaciones ( $y$, así, con la clase de modelos matemáticos) que se han usado para describirlo, en la construcción de una teoría (así, una clase de modelos matemáticos) del último.

6 Ese enunciado, en su libro Metaphysical Foundations of Natural Science, continúa un tema racionalista de Descartes y Leibniz y el mismo se recaptura en el artículo de Duhem, "Theoretical Physics is Mathematical Physics". 
Él califica esto de inmediato, señalando que el hecho de que esto sea en principio posible no implica formalmente ique sea (realmente) posible! En efecto, él dice que el que sea posible o no en cualquier caso dado, no puede decidirse a priori. Añade, y esto suena muy amenazante:

Tiene especial importancia considerar la posibilidad de tal sistema para la totalidad de los objetos de conocimiento. Incluso en este caso, no es posible hacer una decisión a priori. Pero más tarde veremos que cualquier ciencia intersubjetiva, racional, presupone esta posibilidad. (Sec. 13, p. 25)

En otras palabras, podríamos estar a un paso de una deducción trascendental; que en principio ésta sea siempre posible es un argumento muy débil, pero puede reforzarse con la propuesta de que es necesaria sobre el presupuesto o como precondición de la posibilidad misma de la ciencia racional, objetiva, intersubjetiva.

No creo que tengamos que recorrer sus ejemplos y dificultades en las secciones 14-16, pues la propuesta general puede fácilmente apreciarse hoy. $\mathrm{Si}$ el universo o el dominio específico que se considera es invariante bajo ciertas transformaciones -esto es, si exhibe alguna simetría, por abstracta o abstrusa que sea, la descripción no fijará la referencia de manera única. Dado un cuadro científico particular del mundo ( $y$ el de Carnap es muy, pero muy newtoniano o, al menos, aún moderno ${ }^{7}$ tales dudas acerca de la unicidad de la descripción pueden parecer ridículamente escépticas. Pero dados los avances científicos más recientes, no podemos ser tan moderados. Tanto en el caso de la física del espacio-tiempo, con sus isometrías sorprendentes - tan sólo piénsese en los diversos "argumentos de hoyo" acerca del determinismocomo en la mecánica cuántica, con sus simetrías de permutación, tenemos justamente el tipo de simetrías de largo alcance y profundidad por las que Carnap se preocupaba.

Así, Carnap intenta eliminar la necesidad de la ostensión; 'qué sucedería si todo tuviese una única descripción identificadora? Él vacila entre dos maneras de insistir sobre eso: a) que eso es así es una presuposición de la ciencia (supongo que esto significa que lo tenemos que creer sea o no verdadero); b) que hemos de adoptar una ontología en la cual el isomorfismo implique la identidad. Pero, desafortunadamente, ininguna de ellas eliminaría la necesidad de la descripción ostensiva! En cualquiera de esos casos, presumiblemente la ciencia, en caso de tener éxito, nos proporcionaría "mapas" (modelos) con asimetrías extendidas. Pero, aún tendríamos sólo un mapa y tendríamos que localizarnos a nosotros mismos con respecto a eso. La descripción definida

7 Véase "Possible Physical Bases", sec. 62, con su muy obsoleto examen de una base que consta de partículas elementales y campos y de la reconstrucción de las partículas elementales en la física del espacio-tiempo. 
puede haber reemplazado a la ostensión en el nivel teórico, pero ésta vuelve tan pronto como queremos hacer uso de la teoría.

Hay sólo una solución, la solución misma que Carnap temía, esto es, admitir, después de todo, a la subjetividad en la ciencia. Pero, zcuáles, exactamente, son las implicaciones de esta solución? Primeramente, la ciencia aplicada es autónoma; las condiciones de posibilidad de la ciencia aplicada, incluyen más que el conocimiento o la creencia en las teorías y modelos que proporciona la ciencia pura. Pero, ¿qué es este "más"? No es un tipo de hecho, misteriosamente distinto, que no puede codificarse en el mapa. La narración científica puede completarse en el sentido de describir todos los hechos, incluyendo que alguien tenga o no tenga el "extra" que se requiere de él o ella para aplicar una porción particular de ciencia. Es tan sólo que describir el tenerla, ino es un sustituto de tenerla!

Empero, este acto de localizarme a mí mismo con respecto a un mapa/modelo comprende una bipótesis empirica. Para que una persona particular haga una predicción específica contrastable en una situación dada, se requieren dos elementos:

1) Una creencia acerca de lo adecuado del mapa +2 ) autolocalización $\Rightarrow$ predicción empírica.

Aquí, 1) admite más o menos y 2) puede ser más o menos determinada ("Estamos en algún lugar por aquî"). Así que el acto aún se llama propiamente una aseveración, es un acto linguístico y puede "refutarse" (con respecto a creencias generales acerca del mapa, claro está). Daré más detalles acerca de esto en la sección 3, donde nos encontraremos estos actos en un entorno diferente.

Sólo tendremos que admitir un sentido no peyorativo de "subjetivo". Es verdad que esta solución otorga un papel especial a la conciencia en la ciencia. Pero sólo hace eso sobre la premisa de que hay ciencia aplicada, esto es, de que hay uso consciente de la ciencia. La solución no implica formalmente más de la conciencia de lo que está contenido en esa premisa.

\section{Espectro II: pérdida del lenguaje}

Puesto que el acto de localizarme a mí mismo con respecto a un mapa o a un modelo es un acto lingüístico, debemos preguntarnos qué es lo que sucede cuando aplicamos este mismo análisis al lenguaje. (Éste, claro está, fue uno de los grandes proyectos de Quine, aplicar los resultados de la filosofía de la ciencia al lenguaje en el que escribimos la ciencia - y el resultado al que llegó fue la relatividad ontológica. Sin embargo, yo relacionaré los problemas, no con los textos de Quine, sino con otro texto de Carnap.) El segundo espectro susurra que, en algún sentido, nosotros no conocemos nuestro propio len- 
guaje - nosotros no sabemos y, quizás, ni siquiera hay ningún hecho acerca de qué es lo que estamos diciendo y a qué nos referimos.

Aquí está la segunda perplejidad elemental acerca de los mapas que deseo relataros. ¿Qué es exactamente estar perdido? Imaginadme en Taxco y comparadme con un ciudadano nativo de Taxco. Mientras camino por aquí, me pierdo; en algún lugar miro a mi alrededor y no sé en donde estoy. Le pido a este ciudadano que me ayude y ríe, él nunca se ha perdido en Taxco sin importar a dónde vaya. Pero, luego de que me ayuda le pido que me dibuje un mapa de la ciudad y sus alrededores. El mapa es meticuloso, con proporciones muy precisas, su casa al centro y cada prominencia señalada con su propia altura. Me llevo este mapa cuando parto y, durante el siguiente año, busco muchos mapas y atlas en el departamento de libros de referencia de nuestra biblioteca.

Encuentro veintisiete áreas que son exactamente como las que presenta este mapa, de ciudades alrededor del mundo (quizás también en otras galaxias; ésta, después de todo, es una fantasía filosófica). Usando copiadora, tijera y pegamento, produzco veintisiete mapas de esas veintisiete áreas, comparables con su mapa dibujado. $\mathrm{Y}$ al año siguiente, cuando regreso, le muestro estos mapas (quizás, incluso como parte de un mapa mayor, de tal manera de relacionarlos entre sí), aun cuando todos están etiquetados en una lengua extraña que él no puede entender. Le pido que me muestre en cuál de estos veintisiete mapas está él. Por hipótesis, él no puede hacerlo. Así que afirmo que está perdido - iél no sabe en dónde está!

Claro está que a menos que él también sea un filósofo, esto no lo confundirá. Simplemente me dirá que de ninguna manera está perdido y si le doy uno de estos atractivos mapas, él lo reetiquetará para sí mismo y será un mapa muy útil de Taxco. Llamemos a esto el truco de los 27 mapas.

Una ilustración histórica de cómo los mapas pueden reemplazar la realidad en la mente filosófica, pertenece a la filosofía del lenguaje, donde los mapas (modelos, en el sentido del científico más bien que del lógico) ${ }^{8}$ de partes de nuestro lenguaje son "lenguajes artificiales (formales)". El libro de Carnap, La sintaxis logica del lenguaje, se escribió como auxiliar de un gran proyecto, a saber, transformar la filosofía en una lógica de la ciencia y, con ello, en una parte de la sintaxis. "Sintaxis" se refiere aquí a cierta teoría, la teoría de la sintaxis, que aborda el lenguaje viviente, natural, en el que también se formula la ciencia, construyendo sistemas puramente sintácticos como lenguajes artificiales. Siguiendo el procedimiento científico adecuado, éstos se ofrecen como modelos, de complejidad cada vez más amplia e incrementable, de los lenguajes

8 De ahora en adelante se usará "modelo" en el sentido en que lo usa el científico (modelo de un fenómeno o del átomo, el sistema solar, el cosmos, inglés de los Apalaches, etc.), a menos que se especifique de otra manera. Los modelos son estructuras, usualmente estructuras matemáticas. 
naturales. Luego, a la larga izas! desaparece el lenguaje natural y lo reemplaza, in toto, el sistema lingüístico construido.

El lógico holandés, Evert W. Beth (1963), atacó las convicciones silenciosas así como las explícitas de Carnap acerca de la relación entre lenguaje natural y los sistemas lingüísticos construidos por los lógicos. La crítica de Beth se expresa de manera más fácil pensando acerca de esto desde un punto de vista semántico. Una sintaxis tiene muchos modelos (en el sentido del lógico); esto es, podemos interpretarla como un lenguaje capaz de describir muchas estructuras diferentes. Es posible limitar esta diversidad de modelos añadiéndole al sistema sintáctico, además del vocabulario y las reglas de gramática, también axiomas y reglas de deducción. Pero hay un límite a esto que, de la manera más notable, puso de manifiesto el teorema de incompletitud de Gödel.

Beth dramatizó esto con la ficción de otro filósofo, Carnap* que lee $L a$ sintaxis lógica del lenguaje. Él se mantiene de acuerdo con Carnap por mucho tiempo, en el sentido de que admite la sintaxis como un modelo adecuado, aunque parcial, del lenguaje natural y luego considera que los axiomas y las reglas de Carnap formalizan correctamente nuestras propias lógica, aritmética, teoría de conjuntos y demás. Súbitamente ihay un desacuerdo! Carnap exhibe la oración de Gödel que es verdadera si y sólo si la aritmética formalizada es consistente y Carnap* dice, ipero esa oración es falsa! Obviamente, a Carnap le sorprende mucho esta reacción, puesto que él mismo está convencido de que es verdadera, aun cuando no es demostrable a partir de los axiomas.

¿Cómo es esto posible? Bien, tanto esa oración de Gödel como su negación se pueden añadir consistentemente a la aritmética. De ahí que hay al menos dos modelos que podrían guiar nuestras intuiciones con respecto a la verdad y a la falsedad, tal como se expresan en el lenguaje natural. Un modelo (o tipo de modelo) debe estar guiando las intuiciones de Carnap y el otro (o el otro tipo) guía las de Carnap*.

Sin embargo, la forma como he descrito esto es un tanto simplista. Supone que aun cuando el lenguaje natural no coincide con un sistema sintáctico, sí lo hace con una sintaxis suplementada con un modelo o tipo de modelo. Desde el punto de vista de la semántica formal, así es como tiene que describirse. Pero, claro está, eso simplemente renueva el gran proyecto, situando la semántica formal en el papel de la sintaxis.

Desafortunadamente, la misma línea de argumento demolerá ese segundo gran proyecto. Pues supóngase que en la teoría intuitiva de conjuntos describimos una sintaxis más axiomas y su clase de modelos. Si suponemos que éste es un modelo adecuado para el lenguaje mismo en el cual trabajamos en este proyecto de construcción, entonces, en ese modelo, vemos una réplica formal de nuestra teoría de conjuntos. Pero, una vez más, podemos imaginar a Carnap y a Carnap*. Ellos, según parece, concuerdan perfectamente, pues cada uno 
de ellos coincide en que son verdaderos los axiomas de la teoría de conjuntos, tal como allí se hallan formalizados. Pero entonces, a medida que siguen adelante, aparece el desacuerdo súbitamente. Nos enfrentamos al mismo tope que antes, aun cuando esta vez proviene del teorema de Löwenheim-Skolem más bien que del teorema de Gödel. ${ }^{9}$

De cierta manera, nuestra situación fue incluso peor que la de Carnap y Carnap*, pues estábamos escuchando a ambos y concordando con ambos, en la convicción de que entendíamos lo que estaban diciendo -incluso estábamos concordando con ambos. Estábamos convencidos de que estaban diciendo lo mismo exactamente porque estábamos convencidos de que entendíamos sus aseveraciones conjuntas y, ciertamente, eso quería decir que extraíamos un significado único de sus palabras. Entonces, súbitamente, el desastre, el terreno se abre bajo nosotros: ellos siempre entendieron la cuestión de dos maneras, en forma diferente. Así que, entonces icuál fue nuestra comprensión de ella? resultó que ellos hablaban lenguajes diferentes que sonaban igual -así que, ‘en qué lenguaje estábamos escuchándolos? ¿Cuál era nuestro lenguaje?

El carácter lógico del argumento hace que sea muy general. De hecho, no hay tan sólo Carnap y Carnap*, sino $\operatorname{Carnap}^{*}(1), \ldots$, Carnap*(1000),.., Carnap $^{*}(\omega+1), \ldots$, etc. De todos estos posibles lenguajes que suenan igual, ¿cuál es mi lenguaje? No hay forma de decidir y, peor aún, parece que no hay ningún apoyo factual.

Para diagnosticar esta confusión y sacarnos de ella, necesitamos restaurar un sentido más robusto de la realidad. La primera cosa en la que hay que insistir es que si alguien está hablando acerca de tal familia de lenguajes posibles, el valor facial es que en realidad está hablando de un modelo - una multiplicidad matemática de lenguajes formales que en sí mismos, claro está, son sólo entidades matemáticas. Los lenguajes formales son modelos de juegos de lenguaje, pero los únicos juegos de lenguaje reales son los que se jugaron o jugarán, en el pasado, en el presente o en el futuro.

¿De qué son modelos estos lenguajes formales? Aquí debemos distinguir el lenguaje en el sentido de los recursos que tenemos para construir y jugar juegos de lenguaje, de los juegos de lenguaje reales que de hecho se juegan. Los lenguajes formales son modelos de juegos de lenguaje ( $y$ no un modelo de los recursos). ${ }^{10}$

Ciertamente hay una subdeterminación de la evidencia y, quizás, de todos los hechos, por hipótesis que relacionan estos lenguajes formales, qua modelos, con nuestro lenguaje. Los únicos juegos de lenguaje reales son los que

9 Este nuevo Carnap* muy bien podría denominarse Putnam*, en vista de los bien conocidos argumentos de Putnam que se apoyan en el resultado de Löwenheim-Skolem.

10 Véase, además, mi 1986. 
se jugaron o jugarán, en el pasado, en el presente y en el futuro. Desde un punto de vista matemático, esto es muy limitado. Ciertamente, cada uno de ellos se juega, presumiblemente, sólo durante una cantidad finita de tiempo. Así que Quine tiene razón cuando señala que debe haber, entre todas las funciones matemáticas que hay, muchos manuales de traducción adecuados que relacionen, de diversas maneras, nuestro lenguaje (actualizado) con diversos lenguajes formales. Aquí, adecuado quiere decir empíricamente adecuado, esto es, cuadra con todos los fenómenos lingüísticos reales.

Pero supongamos que no hacemos aseveraciones y no formamos creencias que vayan más allá de afirmaciones de adecuación empírica para tales hipótesis. Yo ciertamente no veo eso como un defecto epistémico. Ciertamente significa que dejamos abiertas muchas preguntas acerca de nuestro lenguaje, pero para nada significa que no conozcamos o que no entendamos nuestro lenguaje. Los muchos manuales de traducción son semejantes a los muchos mapas en el truco de los 27 mapas, que ciertamente no establece que el habitante nativo de Taxco está perdido cuando camina alrededor de Taxco.

Hablar de todos los juegos de lenguaje posibles sólo puede equivaler a hablar de todos los elementos de un modelo, una multiplicidad de lenguaje formales. Esto se parece mucho al caso de la perspectiva espacial, pues si deseamos hablar acerca de toda posible perspectiva espacial, nada mejor podemos hacer que, o ciertamente nada diferente de, hablar acerca de todos los puntos en cierto espacio matemático -esto es, hablar acerca de un modelo. ${ }^{11}$

Ciertamente aún hay otras propuestas que hacer aquí, específicamente que tener un lenguaje (o que cierto juego de lenguaje sea mi lenguaje) no puede reducirse a tener conocimiento o creencia "objetiva". Pero no continuaré aquí con esto - creo que se ha desarmado el espectro de que no sabemos qué lenguaje estamos hablando, una vez que distinguimos la realidad de tener un lenguaje, vivir en un lenguaje (que es como vivir en Taxco), de la multiplicidad de "mapas" en el truco de los 27 mapas, en tanto que se aplica al lenguaje.

\section{Espectro III: perdida de la experiencia}

Estudié los dos primeros espectros principalmente como un prolegómeno para el tercero, que encuentro mucho más amenazador. Es el espectro de lo que popularmente se denomina el relativismo Kuhn-Feyerabend. ${ }^{12}$ Esto es

11 De hecho, cualquier intento de hablar acerca de todos los juegos de lenguaje posibles de manera que vaya más allá de esto conduce al tipo de paradojas examinadas en el escrito de Raúl Orayen en este mismo volumen.

12 Eso no significa que Feyerabend o Kuhn lo suscriban o que jamás lo hayan suscrito; el nombre se refiere a la inspiración que floreció donde se oyó. 
pertinente para mí en tanto que este relativismo implica una "pérdida de experiencia", en tanto que disuelve la dicotomía observación/teoría.

Cuando me relaciono con mapas y con lenguajes formales, mis aseveraciones, en efecto, describen dónde estoy y qué lenguaje es mi lenguaje, el juego de lenguaje que hablo. Pero este lenguaje está históricamente condicionado, ha adoptado cierta estructura mediante un desarrollo histórico y, de manera más específica, mediante el desarrollo de teorías científicas (y otras). Si ahora usamos este lenguaje para dar cuenta de mi experiencia, esos informes están estructurados y condicionados por la "figura" de mi lenguaje. No puede ser de otra manera, pues éste es el único lenguaje que yo tengo. Así que, ¿cómo puede ser la experiencia la piedra de toque objetiva para la ciencia y cómo puede la ciencia tener cualesquiera pretensiones de ser diferente de la metafisica? ¿No derrota, la infección teórica del lenguaje, cualquier demarcación entre lo teórico y lo observable?

Mucha gente que se considera retoño intelectual de Hanson, Sellars, Kuhn y Feyerabend, parece haber concluido que una vez que se abandonó el fundacionismo la experiencia sí resultó no ser una piedra de toque objetiva para la ciencia y que el desarrollo de la ciencia no obedece criterios más estrictos que los que obedece la metafísica.

\section{I. La tercera propuesta acerca de los mapas}

Antes de explicar a qué amenaza este tercer espectro, quiero presentar una tercera propuesta sobre los mapas. Cuando les pedí que imaginaran que yo estaba perdido y necesitaba localizarme a mí mismo con respecto a un mapa a fin de proseguir, estoy seguro de que ustedes pensaban en un mapa preciso. Supongamos que el mapa es defectuoso o impreciso, \{eso excluye el acto de autolocalización en él, lo hace imposible o bien inútil? El hecho es, claro está, que todos los mapas que usamos dejan algo que desear y algunos de los más útiles están muy distorsionados. Sólo piensen en los mapas del metro; éstos son imprecisos con respecto a figura y distancia y son precisos sólo con respecto a algunos rasgos topológicos gruesos de la situación. Sin embargo, sin dudar nos localizamos en ellos y encontramos que eso es útil. Piénsese en un mapa del sistema ptolemaico del universo. A pesar de nuestra certidumbre de que ni siquiera es topológicamente correcto, hay una forma correcta y una equivocada de localizarnos en él. En una edición del Paraíso perdido vi un mapa ilustrativo de su universo, con la Tierra en el centro, el Cielo arriba y el Infierno abajo. Ahora, esto me puede molestar sea porque presenta regiones que definitivamente no se encuentran en el espacio-tiempo o, quizás, puedo decir con el Mefistófeles de Marlowe: "iÉste es el Infierno y no estamos fuera de éll" Sin embargo, sé perfectamente bien que hay una forma correcta de localizarme en este mapa, a saber, apuntando a la Tierra en el medio. 
Una forma en la que la filosofia del lenguaje siempre le ha fallado a la filosofía de la ciencia, es en su desapego por el lenguaje defectuoso. Nosotros nos comunicamos en lenguaje defectuoso y sabemos bien que lo hacemos. Sabemos, igualmente bien, que nos localizamos en mapas defectuosos y que los modelos que son más útiles en la ciencia aplicada, generalmente cuadran con los sistemas reales de las maneras más imprecisas que uno pueda imaginar. Si ciertamente es verdad que la ciencia aceptada en el pasado infecta nuestro lenguaje $-y$ yo pienso que, de muchas maneras, esto es verdad-entonces siempre estamos en la posición de escribir nuevas teorías en un lenguaje que es sumamente defectuoso si esas nuevas teorias son correctas. La propuesta se aplica a mapas y a lenguajes por igual; necesitamos y pretendemos tener, precisión sólo en aspectos pertinentes - la imprecisión en otros lugares no vacía los criterios de corrección de autolocalización con respecto a ellos.

\subsection{La imagen científica}

Puesto que espero ser un empirista e intentaré lograrlo, quiero rechazar, con todas mis fuerzas, el relativismo Kuhn-Feyerabend. Para mí, al menos, es un espectro que me dice que mi experiencia está perdida y, ciertamente, que está perdida exactamente porque se perdió la posibilidad de fundamentación epistémica. Pero pienso que este espectro, como los otros, se nutre de confusiones y de una nostalgia mal enfocada.

Cuando escribí The Scientific Image, segui a Sellars y a Feyerabend en la teoría pragmática de la observación. Hice esto sin reflexión, porque era un estudiante de Sellars. (Yo no usaba ese nombre, que introdujo Feyerabend.) Me pareció que esta versión de la observación era una parte central del realismo científico, así que la hice participar de mi oposición. Por esa razón, no se me ocurrió que yo debería argumentar mucho a su favor. Me restringí, principalmente, a la propuesta de que el que podamos o no observar algo es, más o menos, la misma cuestión acerca de si una persona puede funcionar como un detector (aparato de medición) de la presencia de ese tipo de cosa (en el sentido de medición en física). ${ }^{13}$ Claro está que esta teoría tiene, como su artículo más destacado, la nota 1 de "Thesis I" de Feyerabend:

la interpretación del lenguaje de observación está determinada por nuestras teorías y cambia cuando esas teorías cambian.

Yo estaba un poco ciego acerca de cómo esto podría confundir a mis lectores. Esa tesis fue, precisamente, la que dio origen al tipo de relativismo que acabo de describir aquí como eliminando la piedra de toque de la experiencia. Por tanto, ahora permítaseme, primero, recontar la teoría y luego detallarla.

13 Véase The Scientific Image, pp. 14-17, 56-59, 80-81. 


\subsection{La teoría pragmática de la observación: versión inicial}

El paso l en la teoría pragmática de la observación es rechazar el Mito de lo Dado. No hay juicios que sean teórico-neutros, epistémicamente seguros y autoautentificantes. No hay fundamentos de la creencia racional.

Cuando mis estudiantes quieren hablar acerca de informes de observación, les digo que no tomen ejemplos tan artificiales como el "irojo aquí, ahora!” de Russell. Deberían de tomar un ejempo representativo típico, como:

(1) iMirad, el flogisto escapa!

Como lo indicó Feyerabend, un científico del flogisto muy bien podría gritar "iFuego!", pero él mismo, si ha creído y asinilado plenamente la teoría, consideraría eso tan sólo como una forma abreviada de (1). Pierre Duhem ya había señalado que en el laboratorio los informes toman esas formas infectadas de teoría y, felizmente, a partir de entonces, los filósofos redescubrieron esto varias veces (señaladamente circa 1930 y, nuevamente, circa 1960).

El paso 2 es señalar que no aprendemos cómo hacer esos informes de observación mediante entrenamiento en inferencia o en traducción lógica. Más bien, aprendemos condicionando la habilidad de decir esto bajo ciertas circunstancias, así como los perros de Pavlov aprendian a salivar cuando tocaba la campana.

Debemos ser muy cuidadosos para no malentender este paso. Parece similar a otra teoría de "mecanismo perceptual", como la que encontramos en la filosofía tradicional, aunque con aire más "científico" porque se hace referencia a Pavlov. En lugar de esto, este paso es su exacto opuesto, es un rechazo de esas teorías, es insistir en que éstas no son epistemología, sino pseudociencia. (Podemos recordar algunas de ellas: la mente capta la propiedad, luego capta la sustancia y, finalmente, el nexo; entonces, con sus manitas, reunió éstas para hacer una proposición,..., etcétera.)

No, aquí la referencia al condicionamiento sólo tiene una función, mostrar que no hay eslabón necesario entre el contenido del informe de observación y las condiciones a las que responde. El objeto de las narraciones de los mecanismos metafísicos de percepción, era el de asegurar a priori tal eslabón. Pero ése es un error. Nosotros podemos condicionar a una persona para que grite "iBingo!" cuando ve fuego, a que diga "Ahí va una partícula alfa" cuando ve un rastro en una cámara de niebla o a que emita "iOtro platillo volador!" siempre que vea luces en el cielo. Por medio de este condicionamiento - sin que nos importe qué proceso pueda ser- se establece una correlación que, en efecto, hace de la persona un confiable instrumento medidor o detector de las condiciones a las que está condicionado a reaccionar de esa manera.

Feyerabend llamó "pragmática" esta versión debido al papel que le asignó a la interpretación cuando desarrolló estos dos primeros pasos. Un ejemplo 
que parece haber tenido en mente es el siguiente. Supongamos que, en algún momento, la teoría del flogisto tuvo el tipo de hegemonía cultural que adquirió posteriormente y tiene ahora la teoría de la oxidación de Lavoisier. En presencia de fuego la gente gritaba "iFuego!", tanto antes como ahora. En el periodo más temprano, la gente pensaba que, en efecto, eso abreviaba "Estoy en presencia de un escape de flogisto". Nosotros decimos que, también entonces, con esos gritos, la gente informaba de la presencia de fuego -con lo que, claro está, queremos decir oxidación.

Hay una dificultad en esto. Es ciertamente verdadero (usted y yo concordamos) en que la gente típicamente ha gritado "iFuego!" cuando está en la presencia de oxidación. Pero, cuando lo hicieron, no les pareció que estuviesen en presencia de oxidación, sino que estaban en presencia de un escape de flogisto $-\mathrm{y}$ eso es lo que querían decir con sus palabars. Por tanto, hay algo demasiado simplista en la información, mediante una oratio obliqua, de su expresión como: informaron, confiablemente, que estaba sucediendo la oxidación cuando se encontraban en presencia del fenómeno.

A lo mejor, Feyerabend tuvo también otro ejemplo en la mente en el que, quizás, nosotros sólo sabemos que en cierta cultura medieval la gente confrablemente gritaba "Vuur" cuando veían fuego y hemos perdido todo conocimiento de su lenguaje y de sus creencias. Aquí tenemos un problema de traducción radical y quizás Feyerabend pensó que nuestra traducción correcta o, quizás, una admirable de su grito "Vuur" es: "Estoy en presencia de oxidación". Pero esto tiene la misma extrañeza, pues de hecho ('con seguridad?), en nuestra opinión, con la mayor probabilidad ellos no querían decir eso. Pues, para decir eso, deberían de haber tenido el concepto de oxidación, por tanto, algo como la teoría de Lavoisier y parece improbable que lo tuviesen.

Ceguera y perspicacia: la insistencia en el carácter histórico del lenguaje y su infección por las teorías, con la consiguiente "conformación de la experiencia" - el mismo manejo que les dio importancia a los papeles de historia y de interpretación-, aparentemente estuvo aquí acompañado por cierta crueldad con respecto a la interpretación, una disposición a imponer meramente la interpretación hasta llegar al anacronismo. Necesitamos una elaboración más cuidadosa de la noción de "informe de observación".

\subsection{La teoría pragmática de la observación: detalle}

El término "informe de observación" es un término técnico, un trozo de jerga filosófica. La explicación debe respetar el propósito para el que se introdujo y cuadrar con los paradigmas con los se pretendía que cuadrara. Claro está que no necesitamos respetar todas las creencias que puedan tener los filósofos acerca de esos paradigmas. Los más importantes de ellos son ciertas expresiones en el laboratorio o en el observatorio. Los informes que emiten tales institu- 
ciones comunican los datos ya reducidos, resumidos y corregidos mediante métodos estadísticos. Pero esos informes resumidos se basan en informes individuales de observadores entrenados - y ésos son el paradigma de los informes de observación. Sin duda éstos se han enfocado porque los filósofos empiristas ven ahí la piedra de toque última, la línea de fondo entre los criterios de éxito en la ciencia.

Otros ejemplos clasificados con éstos comprenden gritos de “iFuego!” cuando la gente ve fuego y "Rojo aquí, ahora" si han sido especialmente contratados para examinar muestras de color, para buscar manchas de sangre o son esudiantes de Bertrand Russell. Con todo esto en mente, intentemos explicar de nuevo este término técnico.

¿Bajo qué condiciones clasificamos una enisión como un informe de observación? Formulo la pregunta de esta manera para darle plena libertad al papel de nuestras propias creencias que, al menos, es una de las cosas a las que se alude cuando la gente dice que nosotros "interpretamos". Pues, claro está, nosotros clasificamos sobre la base de nuestras propias creencias. (iConsidérese la alternativa!) Esto es inocuo, pues se aplica igualmente a nuestro proceso de clasificar mariposas y minerales.

Al responder la pregunta debemos distinguir entre usos muy estrictos y más sueltos o derivados de "informe de observación". También debemos separar casos en los que ya entendemos las palabras que se usan y en los que no. (Por razón del ejemplo supongo que hoy en día aún entendemos "Está escapando flogisto", aun cuando la tecría del flogisto se haya rechazado desde hace mucho.) Primero el caso central: el sentido estricto, para el caso en el que no entendemos la palabra. Supongamos que la gente $x$ confiablemente (con alta correlación) emite ' $E$ ' bajo ciertas condiciones $f\left({ }^{\prime} E\right.$ '). Un ejemplo de esto, que considero que no es un informe de observación, es que la gente confiablemente grite "iMadre!" en situaciones de peligro mortal.

Condiciones especiales para el caso central:

1) La emisión de ' $E$ ' es sintomática de su propia verdad.

2) Con ocasión de la emisión de ' $E$ ' le parece al emisor que $E$.

3) ' $E$ ' es un informe sobre el acaecimiento de un suceso, proceso o estado de cosas observable (que incluye la presencia de un objeto observable). ${ }^{14}$

4) La emisión de ' $E$ ' desempeña, para el emisor, el papel de una autolocalización, autoadscripción, con respecto a algún mapa, cuadro, modelo o descripción general del mundo. ${ }^{15}$

14 Añadí esta condición tras los agudos comentarios de Ernest Sosa.

15 Esto es lo que es correcto del "Rojo aqui, ahora" de Russell, esto es, los informes de observación son deícticos, son actos de autolocalización. 
Creo que 3) hará fruncir las cejas de la mayoría, pero permítaseme, primero, hacer un comentario sobre el conjunto total de las condiciones y luego sobre cada condición por separado.

Estas condiciones no tienen ningún sentido para los casos en los que aún no entendemos ' $E$ '. Más aún, ellas son muy estrictas. Sin embargo, a lo más es un ejercicio simple construir los sentidos derivados que debilitan estas condiciones, de tal manera de sancionar tales usos derivados tal como se ejemplifican en:

a) Los informes de observación de Pedro son confiables pero están formulados en un lenguaje infectado por una teoría falsa.

b) Los gritos ininteligibles de Pedro son, en efecto, informes de observación del tipo...

c) Los informes de observación de Pedro son inteligibles y están infectados sólo por teorías que nosotros también creemos, pero muy poco confiables.

d) Las emisiones “iFuego!" de Pedro, son informes de observación acerca de fuego, pero sólo cuando no se embriaga, lo que en su caso es raro.

“iEscapa flogisto!”, tal como se decía en la época de gloria de la teoría del flogisto, probablemente habríamos de describirla hoy como un ejemplo de tipo a). Dejaré, como ejercicio, la formulación de estos sentidos y ejemplos derivados. ${ }^{16}$

En 1), se reinstituye el eslabón condición-contenido, pero nótese bien que no como a priori, "interno", "lógico en general" o lo que sea, sino en la forma de una condición factual de alta correlación (implicada por nuestras creencias acerca de la actuación del enisor). Claramente podemos considerar que se satisface esta condición para aseveraciones altamente teóricas, pero sólo cuando creemos suficientemente mucho de la teoría.

Aquí, claro eștá, el uso de "síntoma" es postfundacionista; nosotros no requerimos certeza, sólo alta correlación. Pero esto también necesita formularse de manera cuidadosa. Esperamos, por ejemplo, que sea alta la probabilidad condicional ( $x$ está en presencia de fuego dado que $x$ grita "iFuego!"). Nosotros no añadimos que hay una alta probabilidad de que grite "Fuego" cuando está en presencia de fuego - con frecuencia puede encontrarlo sin importancia. Pero sí queremos añadir que es baja la probabilidad condicional ( $x$ grita "iFuego!" dado que $x$ no está en presencia de fuego) —el síntoma debe separar la condición de sus rivales. Un examen más detallado de este tipo de requisito

16 Importante para algunos usos derivados es la idea de clasificación condicional, esto es, clasificación sobre el supuesto de (por ejemplo, de teorías que cree el emisor). Esto puede producir alguna complejidad (piénsese en la obligación condicional y en la probabilidad condicional). 
debería surgir de los tratamientos detallados de la noción general de medición en los fundamentos de la mecánica cuántica.

La propuesta 2) requiere que la persona que emita ' $E$ ' no esté tan sólo repitiendo como perico, sino realmente informando de cómo le parece en ese momento. Hay un importante sentido derivado, menos estricto, que no está incluido en el ejemplo anterior. En provecho de la eficiencia, como participante en una investigación, un investigador puede hundirse a fondo en el cuadro del mundo de teorías de trasfondo aceptadas - suspendiendo su incredulidad o su agnosticismo con respecto a algunas de sus implicaciones. Entonces, esta persona no está sólo hablando sino pensando y respondiendo fácilmente bajo el supuesto de esas teorías de trasfondo. Considero que éste es un fenómeno común y prevaleciente, en efecto, no sólo en la investigación científica sino virtualmente en todo tipo de diálogo civilizado.

La propuesta 3) no tiene sentido a menos que la pregunta acerca de si son observables los sucesos descritos por ' $E$ ', sea independiente de la pregunta acerca de si su emisión es un informe de observación. Puesto que ejemplos típicos de ' $E$ ' son oraciones en lenguaje altamente infectado de teoría, esto requiere que la distinción observable/inobservable sea independiente de teoría. Esto lo abordaré en la siguiente subsección.

La propuesta 4) la considero la más importante, a la par de 1). Pues la importancia de los informes de observación se deriva, en gran parte, de que sean (en términos de Sellars) "movidas de registro lingüístico". La primera de estas condiciones pueden satisfacerla ejemplos como "Llueve más de tres veces al año en Indianapolis" u "O bien llueve o no llueve".

Todo nuestro examen de autoadscripciones autolocalizantes fue, a fortiori, un examen de informes de observación. Tienen, como texto completo, una aseveración deíctica que, en esa ocasión, localiza al hablante en alguna parte definida de su propio cuadro del mundo general, "objetivo". ${ }^{17}$

Finalmente, consideremos en qué sentido es "relativo" o dependiente de teoría el status del informe de observación. Lo que podemos decir en este punto (esperando el examen de la condición 3)) es que no. Imaginemos, por ejemplo, que Pablo cree que:

5) A Pedro le parece que el flogisto está escapando.

Las creencias de Pablo —quien aquí puede estar clasificando las emisiones de Pedro como informes de observación- no entran en la verdad de las condiciones de 5). El que 5) sea verdadera o no, para nada depende de Pablo. Claro está que 5) no será verdadera a menos que Pedro tenga el concepto de

17 Me doy cuenta de que estoy dejando esto algo metafórico analógico; no es literalmente verdadero que llevemos con nosotros nuestra opinión "general" en la forma de una representación que la codifica, como un cuadro o un mapa. Pero, para los propósitos actuales, podemos, razonablemente, representarnos como haciéndolo. 
flogisto, así que la vida mental de Pedro entra en esas condiciones de verdad - esto no es sorprendente, puesto que 5) es acerca de Pedro:

No hay ningún sentido no trivial en el que 5) sea dependiente de teoría o relativo a una teoría. A esta propuesta para nada le afecta el hecho de que Pablo no pueda creer 5) -y, por tanto, pueda no ser capaz de clasificar cierta emisión como un informe de observación- a menos que Pablo tenga el concepto de flogisto. (Piénsese nuevamente en la clasificación de mariposas o de minerales.)

Empero, dadas las posibles diferencias en creencias y conceptos entre Pablo (el clasificador) y Pedro (el emisor), es mucho más fructífero centrarse en la clasificación hecha por una persona en particular (científicos, la comunidad científica) que considerar este tema en abstracto. Hay, claro está, posiciones filosóficas conforme a las cuales no hay ningún apoyo factual acerca de si le pareció a Pedro que el flogisto estaba escapando (si se refería al flogisto cuando dijo "flogisto" o si, supongo, se refería a sí mismo cuando dijo "yo"). Sólo me preocupa señalar que nada en la teoría pragmática de la observación, hasta aqui, apoya tales posiciones aun cuando, claro está, de alguna manera, cada una podría injertarse en la otra.

\subsection{El carácter empírico de la observación}

Como dije, el término "informe de observación" es un término técnico de filosofía. La palabra "observar", por otra parte, tiene un uso común, más o menos el mismo que el de "percibir". Lo mismo es verdad de la palabra "observable" (aun cuando también se le han dado usos técnicos, por ejemplo, en la mecánica cuántica). Considero que en la discusión filosófica se pretende que tenga su uso común, a menos que se indique algo diferente.

Este término, "observable", es muy similar a otras tantas palabras comunes, como "portátil" y "frágil". Son, por así decir, térninos antropocéntricos pues se refieren a nuestras limitaciones. No son, sin embargo, personacéntricos; las computadoras laptop son portátiles y las copas de vino son frágiles, aun cuando algunas personas sean demasiado débiles como para cargar las unas o romper las otras. Las limitaciones a las que se refieren son del organismo humano. Hasta ahora, ni siquiera los filósofos han sugerido que la demarcación de lo frágil haya cambiado tras el desarrollo de instrumentos tan sofisticados como la almádena. Empero, quiero ir más lejos e insistir también en que la distinción observable/inobservable no es, en ningún sentido importante, relativa a una teoría o dependiente de una teoría.

Para precisar nuestras preguntas, considérense los siguientes enunciados:

1) La emisión de $x$, “iEl flogisto está escapando!”, fue un informe de observación. 
2) $x$ observó que el flogisto estaba escapando.

3) $x$ observó un escape de flogisto.

Ninguna de éstas puede identificarse con ninguna de las otras. Más aún, 1) no implica 2) o 3) y 3) tampoco implica 2). Un informe de observación es sólo sintomático de su propia verdad y los síntomas no garantizan más que alta probabilidad. Por tanto, si se emitió el informe de observación, no se sigue que realmente se observó el fenómeno del que se informa.

Hay buenas razones para mantener este corte terminológico. Nosotros deseamos citar el informe de observación como evidencia de que sucedió cierto fenómeno. Eso significa que necesitamos estar muy confiados de que hubo tales informes de observación, antes de que hayamos establecido nuestra conclusión de que hubo cualesquiera fenómenos semejantes. Pero 2) no añade mucho a 1), en caso de que algo añada, fuera de apoyar la veracidad de 1). Además, si l) y 2) son ambos verdaderos, entonces ciertamente lo es 3 ).

Pero, ‘hay algún tipo de dependencia teórica en cualquiera o en todos ellos?

El enunciado 2) no puede ser verdadero a menos que, en esa ocasión, $x$ pudiese darnos el informe de observación "iEl flogisto escapa!" Esto requiere que le parezca que el flogisto está escapando, lo que requiere una creencia en el flogisto y, aún más, el concepto de flogisto.

Pero 3) es muy diferente. Ni usted ni yo creemos en el flogisto. Pero, por un momento, podemos suponer que es verdadera la teoría del flogisto. Conforme a esa suposición, todos los fuegos que vemos son escapes de flogisto y, por tanto, aún conforme a esa suposición, vemos escapes de flogisto. Sin embargo, esta suposición para nada implica formalmente que creamos en la teoría del flogisto o incluso que tengamos el concepto de flogisto. Por tanto, el que 3) sea verdadera o no es muy independiente de las teorías o conceptos que tenga $x$.

Permítaseme también reformular este argumento crucial de otra manera. Desde mi punto de vista actual, posterior a Lavoisier, son satisfactibles conjuntamente las siguientes tres premisas: la primera premisa es la teoría del flogisto, la segunda es que la gente ve fuegos y la tercera es que nadie ha oído o entiende la teoría del flogisto. Sólo la segunda premisa es verdadera, pero mi conclusión se sigue por el hecho de que aquéllas son conjuntamente satisfactibles. ${ }^{18}$

Por tanto, sería muy erróneo intentar explicar 3), y hacerlo, en términos de los reportes de observación o de los juicios perceptuales que la gente puede alcanzar. Es sólo bajo ciertas condiciones especiales que los dos se conectan y

$18 \mathrm{Mi}$ argumento presupone que " $x$ ve $y$ " es extensional. Mi argumento a favor de esto usa el ejemplo del nativo filipino de la edad de piedra (The Scientific Image, p. 15). 
esas condiciones son meramente hechos contingentes. Esto realmente se sigue de la ausencia de cualquier posible eslabón condición-contenido a priori.

La demarcación de lo que es observable y lo que es inobservable se refiere no a 2), sino a 3). La cuestión de las condiciones bajo las que son verdaderos enunciados tales como 3 ) no es una cuestión filosófica, sino empírica. Si alguien intenta forjar opiniones acerca de qué es exactamente lo que es observable, yo lo animaría a que se apoyase en la fisiología, la psicología y la ciencia empírica en general y que para nada les preguntase a los filósofos.

Claro está que, entonces, hemos de esperar que nuestras opiniones acerca de lo que es observable cambiarán a medida que cambie la ciencia. Pero eso no significa que también cambia lo que es observable. El reciente artículo de Laudan y Leplin (1991) cae exactamente en esa confusión. Ellos, de manera muy correcta, señalan que lo que consideramos como observable no es constante a lo largo de la historia de la ciencia. Pero concluyen que la línea entre lo que es y lo que no es observable ha cambiado conjuntamente con ella.

Bueno, nuestra opinión sobre la cantidad de agua presente en Marte tampoco es constante a lo largo de la historia de la ciencia. Sin embargo, la masa de agua en Marte no ha estado cambiando junto con este cambio de opinión. Nuestros juicios de adecuación empírica de las teorías variará, claro está; pero el que esas teorías sean empíricamente adecuadas - al igual que el que sean o no verdaderas - es una característica que no pierden cuando empezamos a pensar de manera diferente.

\subsection{Una interrogante acerca de política epistémica}

Y aún el espectro del llamado relativismo Kuhn-Feyerabend no ha desaparecido. Pues, después de todo, la única entrada para nuestras políticas o decisiones epistémicas serán nuestras opiniones y no los hechos mismos. Probablemente, la propuesta más contenciosa en The Scientific Image fue mi admisión de que la ciencia nos permite no creer las teorías que aceptamos, sino creer sólo que son empíricamente adecuadas. Según yo, la ciencia no requiere más creencia que ésa (aun cuando, claro está, usted puede creer más si lo desea, por cualesquiera razones de satisfacción personal que pueda reunir).

Esta distinción se funda en la independencia de teoría de la adecuación empírica, esto es, de la distinción observable/inobservable. Pero cuando alguien intenta poner en marcha esa política, su entrada, claro está, no es la separación en la naturaleza de los objetos y los procesos observables e inobservables, sino su opinión acerca de esa demarcación. Esta opinión está históricamente condicionada por el lenguaje en el cual él formula todas sus opiniones, incluyendo sus informes de observación -y ese lenguaje está infectado por teorias en las que él cree y que muy bien pueden ser falsas. Así, en la práctica, la prosecución y realización correcta de esta política epistémica 
empirista, será fuertemente perspectivista y depende del carácter histórico del lenguaje del científico o de la comunidad científica en cuestión.

Pero, tras las distinciones que he hecho, esto difícilmente puede sonar como un relativismo debilitante. Es verdad que cuando yo estoy formando y cambiando mi opinión, yo factorizo en los que clasifico como mis propios informes de observación - y éstos no son fundamentos epistémicamente seguros, están llenos de riesgo empírico. Pero, ¿cuál sería la alternativa? Si no le damos entrada a una nostalgia por los fundamentos, no hay alternativa. $\mathrm{Y}$, si no hay alternativa, no se puede decir muy bien que tengamos algún defecto en nuestra vida epistémica debido a eso.

Aquí hemos de distinguir entre dos casos. Cuando se presenta una nueva teoría y podemos trazar una distinción para nosotros entre que sea verdadera y que sea empíricamente adecuada, entonces tenemos una elección. Podemos, entonces, seguir la política de aceptar sin creer. Éste es el primer caso.

Pero la estructura de nuestro lenguaje puede ser tal que no podamos hacer esta distinción y, por tanto, no tenemos tal elección. Esto significa que, como lo hemos visto, la nueva teoría es sólo acerca de lo que es observable y no le atribuye estructura inobservable al mundo. El supuesto problema es, ahora, que este juicio de nuestra parte se deriva de teorías que infectan nuestro lenguaje y, con ello, nuestras opiniones acerca de lo que es observable -y esas teorías infectantes podrian estar equivocadas.

$\mathrm{Si}$, genuinamente, aquí no tenemos elección (entre mera aceptación y aceptación con creencia plena), entonces esas teorías infectantes no tienen nombre y nosotros somos incapaces de concebir algo que les sea contrario. Ningún ejemplo estándar de un paradigma científico jamás alcanzó ese status. La física de Newton reinó por 200 años y no lo alcanzó. Ahora estamos a 500 años de la reavivación renacentista de la teoría atómica y ésta aún no ha logrado ese status. Más todavía, el espectro dice que puede haber tales teorias infectantes y ellas pueden ser falsas $o$, al menos, pueden ser contrarias a las teorías que infectan de igual manera el lenguaje de otras comunidades históricas.

Bueno, el espectro del relativismo Kuhn-Feyerabend se ha reducido ahora a esta preocupación, que yo estoy obstaculizado o condicionado (en algún sentido peyorativo) por posibilidades que no puedo concebir. Sólo los fundamentos cartesianos podrían salvarme de tal destino y no es posible ningún fundamento semejante. Cualquier patrón de racionalidad que haga esto un defecto es un patrón que ningún humano podría satisfacer. Concluyo que también este espectro es sólo parte del vértigo que nos embargó cuando finalmente abandonamos la búsqueda de fundamentos absolutamente seguros. 
Bibliografía

Beth, E.W., 1963, "Carnap's Views on the Advantages of Constructed Systems Over Natural Languages in the Philosophy of Science", en Schilpp, 1963, pp. 469-502. Carnap, R., 1928, Der Logische Aufbau der Welt (1928), trad. al inglés de R.A. George, The Logical Structure of the World, University of California Press, Berkeley, 1967.

—, 1934, Logische Syntax der Sprache, trad. al inglés de A. Smeaton, The Logical Syntax of Language, Kegan Paul, Londres, 1937.

Feyerabend, P., 1958, "An Attempt at a Realistic Interpretation of Experience", Proceeding of the Aristotelian Society, reimpreso en Feyerabend, 1981.

—, 1981, "Realism, Rationalism and Scientific Method", Philosophical Papers I, Cambridge University Press, Cambridge.

Laudan, L. y J. Leplin, 1991, "Empirical Equivalence and Underdetermination", Journal of Philosophy, no. 88, pp. 449-472.

Putnam, H., 1983, "Realism and Reason", Philosophical Papers III, Cambridge University Press, Cambridge.

Schilpp, P.A. (comp.), 1963, The Philosophy of Rudolf Carnap, Open Court, La Salle (III.).

van Fraassen, B., 1980, The Scientific Image, Oxford University Press, Oxford.

_-, 1986, "The World We Speak Of, and the Language We Live In", Philosophy and Culture, Actas del XVII Congreso Mundial de Filosofia (Montreal, 1983), Editions du Beffroi, Montreal. 